\title{
EXPERIMENTAL INVESTIGATION OF A W-BAND GYROKLYSTRON AMPLIFIER
}

\author{
M. Blank, B.G. Danly, B. Levush, Naval Research Laboratory, Washington, D.C. 20375 and \\ P.E. Latham, Omega P, Inc., New Haven, CT 06520
}

\section{Abstract}

A four cavity W-band gyroklystron amplifier experiment is currently underway at the Naval Research Laboratory. The gyroklystron has produced $67 \mathrm{~kW}$ peak output power and $28 \%$ efficiency in the $\mathrm{TE}_{011}$ mode with a $55 \mathrm{kV}, 4.3 \mathrm{~A}$ annular electron beam. The full width half maximum (FWHM) bandwidth is greater than $460 \mathrm{MHz}$. Small signal and saturated gains of $36 \mathrm{~dB}$ and $29 \mathrm{~dB}$, respectively, have been observed. The amplifier is zero drive stable and the limiting oscillation is the $\mathrm{TE}_{011}$ operating mode in the output cavity. Experimental results are in good agreement with theoretical predictions.

\section{INTRODUCTION}

The continuing need for high power sources of millimeter wave radiation for such varied applications as high resolution radars, linear accelerators, and communications, has led to extensive research on gyroklystron amplifiers. Much like a conventional klystron, the gyroklystron consists of several resonant cavities separated by drift sections cut-off to the operating mode. As evidenced in numerous experiments, the interaction of the beam with the trapped mode in the cavity, based on the electron cyclotron maser instability, can reliably and efficiently generate high power, moderate bandwidth electromagnetic radiation at microwave and millimeter wave frequencies. For example, a three cavity C-band gyroklystron amplifier produced $54 \mathrm{~kW}$ peak output power and $30 \%$ efficiency in the $\mathrm{TE}_{101}$ at $4.5 \mathrm{GHz}$ [1]. The saturated gain was $30 \mathrm{~dB}$ and the FWHM bandwidth was $0.4 \%$. A three cavity Xband gyroklystron achieved $16 \mathrm{~kW}$ peak output power $45 \%$ efficiency with a $1 \%$ bandwidth [2]. Fundamental and second harmonic two cavity gyroklystron amplifiers at $9.87 \mathrm{GHz}$ and $19.7 \mathrm{GHz}$, designed as drivers for linear colliders, achieved peak output powers of $20 \mathrm{MW}$ and 30 MW, respectively, with efficiencies near 30\% [3,4]. A two cavity Ka-band gyroklystron, developed for radar applications, produced $750 \mathrm{~kW}$ at $35 \mathrm{GHz}$ in the $\mathrm{TE}_{021}$ mode at $24 \%$ efficiency [5]. In W-band, a pulsed four cavity gyroklystron amplifier achieved $65 \mathrm{~kW}$ peak output power at $26 \%$ efficiency with $300 \mathrm{MHz}$ bandwidth [6]. A continuous wave version of the device demonstrated $2.5 \mathrm{~kW}$ average output power. The goal of the present work is to enhance the bandwidth of the Wband gyroklystron amplifier while maintaining high efficiency, peak output power, and gain.

\section{THEORY AND DESIGN}

This paper presents an experimental study of a four cavity W-band gyroklystron amplifier operating in the $\mathrm{TE}_{011}$ mode near the fundamental cyclotron frequency. The circuit consists of a drive cavity, two idler cavities, and an output cavity. The circuit was designed with a timedependent version of the non-linear code MAGYKL [7]. The wave equation solved in MAGYKL is given by

$$
\frac{d a}{d t}+\left(\frac{1}{2}+i \Delta_{\omega}\right) a=-\frac{Q I_{b}}{2 \omega W_{E M}} \int d \xi \frac{c}{\omega}\left\langle\frac{v_{p e r p} \cdot \boldsymbol{E}_{c} e^{-i \omega t}}{v_{z}}\right\rangle
$$

where $a$ is the complex amplitude of the fields, $\Delta_{\omega}=\mathrm{Q}\left\{\operatorname{Re}\left\{\omega_{\mathrm{c}}\right\}-\omega\right\} / \omega$ is the normalized frequency shift, $Q$ is the quality factor of the cavity, $\omega$ is the drive frequency, $\omega_{c}$ is the cold resonant frequency, $I_{b}$ is the beam current, $W_{E M}$ is the stored energy, $c$ is the speed of light, $v_{\text {perp }}$ and $v_{z}$ are the perpendicular and axial electron velocities, $\boldsymbol{E}_{c}$ is ite cold cavity electric field, and $t$ is the normalized time $\omega / Q$.

In the formulation, the cavities are modeled by a series of straight uniform sections with abrupt discontinuities at the boundaries. The fields in each section, expanded as a radial series of TE, TM, and TEM modes, are determined through a scattering matrix solution [8]. Theoretical studies have shown that it is necessary to include many modes in the field description to correctly predict the resonant frequency of the cavity. In order to accurately predict the bandwidth of the amplifier, the formulation detailed in reference [7] was modified to include a frequency dependent drive power, as dictated by the resonant frequency and $\mathrm{Q}$ of in input cavity. The theoretical model was used to design the interaction circuit and determine the parameters of each cavity, which are summarized in Table 1.

\begin{tabular}{|l|ccc|cc|}
\hline & \multicolumn{3}{|c|}{ Design } & \multicolumn{2}{c|}{ Cold Test } \\
\cline { 2 - 6 } & $\mathrm{L}(\mathrm{cm})$ & $\mathrm{f}_{0}(\mathrm{GHz})$ & $\mathrm{Q}$ & $\mathrm{f}_{0}(\mathrm{GHz})$ & $\mathrm{Q}$ \\
\hline cavity 1 & 0.43 & 93.00 & 125 & - & - \\
cavity 2 & 0.50 & 93.52 & 175 & 93.56 & 130 \\
cavity 3 & 0.50 & 92.89 & 175 & 93.02 & 128 \\
cavity 4 & 0.80 & 93.18 & 300 & 93.21 & 299 \\
\hline
\end{tabular}

Table 1 Summary of cavity parameters. 
The drive power is coupled into the circuit through a coaxial cavity [9]. A single cylindrical waveguide excites the $\mathrm{TE}_{411}$ mode of the outer cavity. Power is then coupled from the $\mathrm{TE}_{411}$ mode in the outer cavity to the $\mathrm{TE}_{011}$ mode in the inner cavity through four slots positioned symmetrically around the azimuth of the cavity. The coaxial cavity was analyzed with the High Frequency Structure Simulator (HFSS) and found to have a resonant frequency of $93.0 \mathrm{GHz}$ and a diffractive $\mathrm{Q}$ of 150. HFSS simulations also show that $75 \%$ of the energy is stored in the $\mathrm{TE}_{411}$ mode and $25 \%$ is stored in the $\mathrm{TE}_{011}$ inner cavity mode. Thus, $6 \mathrm{~dB}$ of drive power arriving at the input cavity is stored in the outer cavity and is not available for interaction.

Because the second and third cavities are terminated by drift sections that are cut-off for the $\mathrm{TE}_{011}$ mode at $93 \mathrm{GHz}$, the diffractive Q's are quite large. The design Q's of 175 are achieved through dielectric loading of the cavities. The output cavity consists of a $0.8 \mathrm{~cm}$ straight section, followed by an iris which is cutoff to the $\mathrm{TE}_{011}$ mode at $93 \mathrm{GHz}$, and a 5 degree linear uptaper to the collector radius. The wave is coupled out diffractively and there is no ceramic loading the output cavity. The first and second cut-off drift lengths are loaded only by the dielectrics on the upstream walls of the idler cavities, and the drift length separating the penultimate cavity and output cavity is unloaded.

\section{EXPERIMENTAL RESULTS}

The circuit was built and cold tested on a vector network analyzer. The cavities were excited and sampled through two $0.075 \mathrm{~cm}$ diameter holes positioned 180 degrees apart in the side wall. The transmission spectra for the idler and output cavities are shown in Fig. 1 and the measured resonant frequencies and Q's are summarized in Table 1.

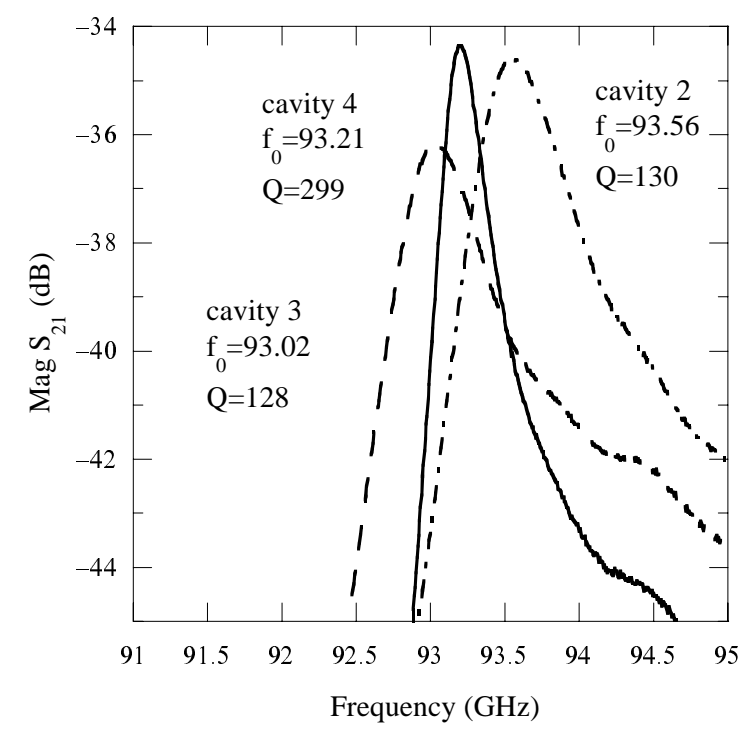

Figure 1 Cold test transmission spectra for cavity 2 (dashed-dot line), cavity 3 (dashed line) and cavity 4 (solid line).

Upon completion of the cold test, the circuit was installed in the test stand. Figure 2 shows a schematic of the gyroklystron amplifier experiment. A $4 \mathrm{~A}, 55 \mathrm{kV}$ annular electron beam is produced by a double anode magnetron injection gun. The magnetic field at the cathode, which is nominally $1.5 \mathrm{kG}$, can be varied to control the beam velocity ratio, $\alpha$. The beam is adiabatically compressed as it enters the region of high magnetic field generated by the $4 \mathrm{~T}$ superconducting magnet. The four cavities of the gyroklystron circuit are positioned in a region of constant magnetic field. The output cavity tapers up to the collector, which is followed by a quartz vacuum window. A conically shaped, water backed teflon load is positioned on the atmosphere side of the vacuum window. The temperature rise of the water is used to measure the average rf power. The frequency of the input and output rf signals are measured with a spectrum analyzer. The drive power is supplied by a 1 $\mathrm{kW}$ peak power EIO, which is mechanically tunable from approximately $92.5 \mathrm{GHz}$ to $95.5 \mathrm{GHz}$. The EIO provides pulses up to 2 microseconds in duration with a duty cycle up to $1 \%$. The beam and EIO are typically pulsed at 250 $\mathrm{Hz}$ for an rf duty cycle of $0.05 \%$.

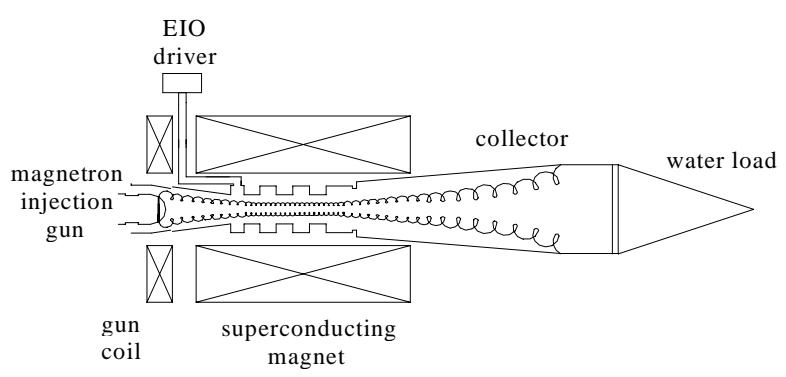

Figure 2 Schematic of the experimental test stand.

Figure 3 shows the measured and predicted output power and efficiency as a function of frequency for the amplified $\mathrm{TE}_{011}$ mode. A peak saturated output power of $67 \mathrm{~kW}$, corresponding to $28 \%$ efficiency, was achieved with a $55 \mathrm{kV}, 4.3 \mathrm{~A}$ electron beam. The FWHM bandwidth is greater than $460 \mathrm{MHz}$. The input power, measured at the output of the EIO, was $87 \mathrm{~W}$, which gives $29 \mathrm{~dB}$ saturated gain. For the theory curve in Fig. 3, the experimentally determined values of cold resonant frequencies and Q's for the idler and output cavities were used. The HFSS predictions of the drive cavity resonant frequency and $\mathrm{Q}$ were assumed. The experimental values of beam voltage, beam current, and magnetic field in the 
interaction circuit were used. The beam $\alpha$ was taken to be 1.5 , and the perpendicular velocity spread was assumed to be $9 \%$, values that were obtained through a combination of modeling and empirical determination. As shown in Fig. 3, the theoretical predictions are in good agreement with experimental data.

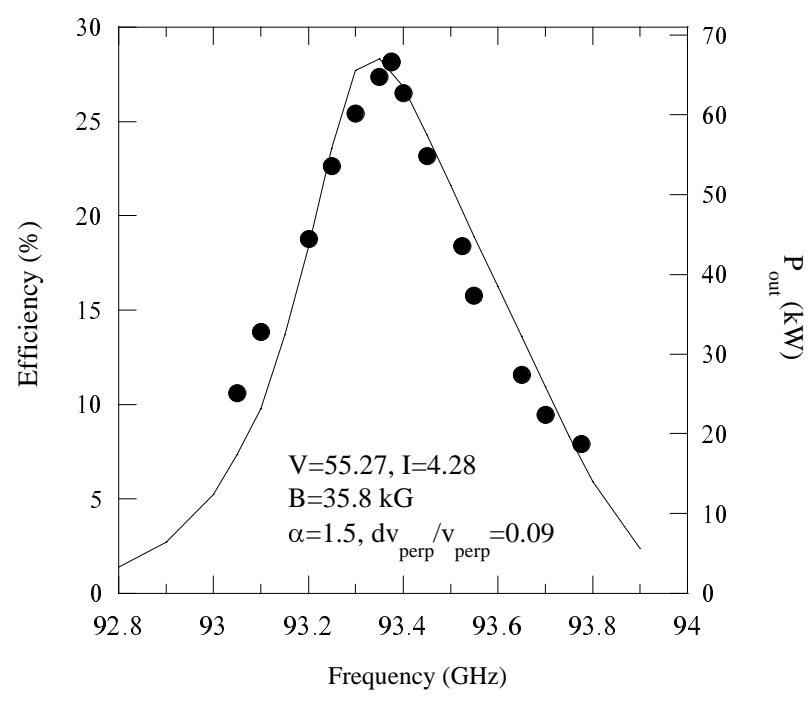

Figure 3 Measured values (filled circles) and theoretical predictions (solid curve) of peak output power and efficiency.

The limiting oscillation in the circuit was found to be the $\mathrm{TE}_{011}$ operating mode in the output cavity. The start oscillation current was measured and the results are shown in Fig. 4.

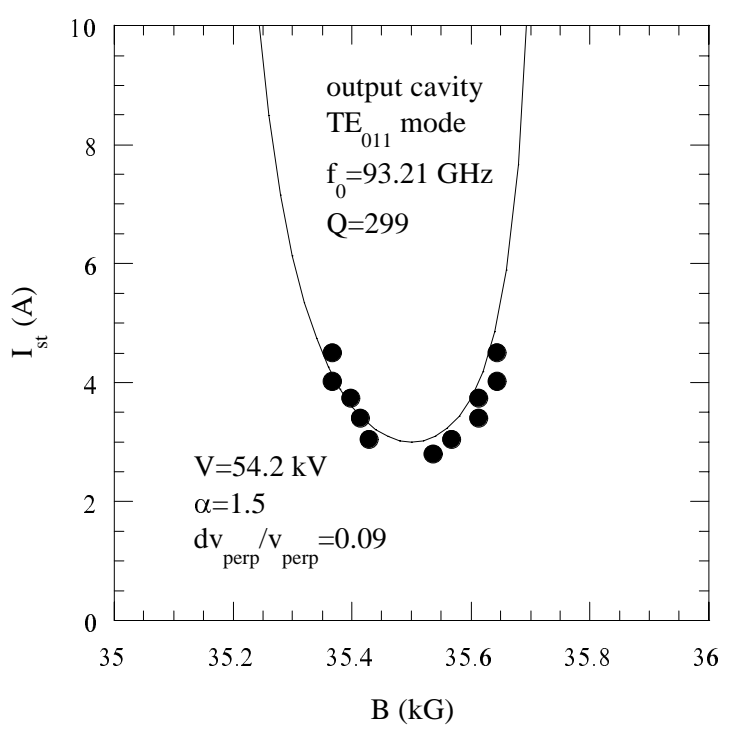

Figure 4 Measured values (filled circles) and theoretical prediction (solid curve) for start oscillation current of the $\mathrm{TE}_{011}$ mode in the output cavity.
The measured data points, indicated by the filled circles, are compared with the theoretical start current,

shown with the solid line. In the theory, the measured values of beam current, cold resonant frequency, and cold cavity $\mathrm{Q}$ were used. The beam $\alpha$ was taken to be 1.5 , and the perpendicular velocity spread was assumed to be 9\%. As shown in Fig. 4, the experimental data and theoretical predictions are in good agreement.

\section{SUMMARY}

In summary, a four cavity W-band gyroklystron amplifier circuit was designed, built, and tested. Peak output powers of $67 \mathrm{~kW}$, corresponding to $28 \%$ efficiency, were achieved in the $\mathrm{TE}_{011}$ mode with a $55 \mathrm{kV}$, 4.3 A electron beam. The FWHM bandwidth is greater than $460 \mathrm{MHz}$. The small signal and saturated gains are $36 \mathrm{~dB}$ and $29 \mathrm{~dB}$, respectively. The circuit is zero drive stable and the limiting oscillation is the $\mathrm{TE}_{011}$ operating mode in the output cavity. The experimental data is in good agreement with predictions of theory using calculated values of beam velocity ratio and velocity spread. Future experiments will focus on increasing bandwidth through more aggressive stagger tuning and reduced output cavity $\mathrm{Q}$.

\section{ACKNOWLEGEMENTS}

The authors would like to thank M. Barsanti, F. Robertson, B. Sobocinski, and M. Ngo for their technical assistance. This work was supported by the Office of Naval Research. The computational work was supported in part by a grant of HPC time from the DoD HPC Center NAVO.

\section{REFERENCES}

[1] W.M Bollen, A.H. McCurdy, B. Arfin, R.K. Parker, A.K. Ganguly, IEEE Trans. Plasma Sci. 13, 417 (1985).

[2] E.V. Zasypkin, M.A. Moiseev, E.V. Sokolov, V.K. Yulpatov, Int. J. Electron. 78, 423 (1995).

[3] W.G. Lawson et al., Phys. Rev. Lett. 67, 520 (1991).

[4] H.W. Matthews et al., IEEE Trans. Plasma Sci. 22, 825 (1994).

[5] E.V. Zasypkin, M.A. Moiseev, I.G. Gachev, I. I. Antakov, IEEE Trans. Plasma Sci. 24, 666 (1995).

[6] I.I. Antakov, E.V. Zasypkin, E.V. Sokolov, Conf. Digest of the Eighteenth Intl. Conf. On Infrared and Millimeter Waves, Proc. SPIE 2104, 166 (1993).

[7] P.E. Latham, W. Lawson, V. Irwin, IEEE Trans. Plasma Sci 22, 804 (1994).

[8] J.M. Neilson, P.E. Latham, M. Caplan, W. Lawson, IEEE Trans. Microwave Theory Tech. 37, 1165 (1989).

[9] G.S. Park, C.M. Armstrong, R.H. Kyser, J.L. Hirshfield, R.K. Parker, Int. J. Electronics 78, 983 (1995). 